\title{
Neurophysiological changes during insulin-induced hypoglycaemia and in the recovery period following glucose infusion in Type 1 (insulin-dependent) diabetes mellitus and in normal man
}

\author{
G. Tallroth ${ }^{1}$, M. Lindgren ${ }^{2}$, G. Stenberg ${ }^{2}$, I. Rosen ${ }^{2}$ and C-D. Agardh ${ }^{1}$ \\ Departments of ${ }^{1}$ Internal Medicine and ${ }^{2}$ Clinical Neurophysiology, University Hospital, Lund, Sweden
}

\begin{abstract}
Summary. Hypoglycaemia (median venous blood glucose $1.8 \mathrm{mmol} / 1$; range $1.6-2.3$ ) was induced by an intravenous infusion of regular insulin in eight patients with Type 1 (insulin-dependent) diabetes mellitus (age $28.0 \pm 7.4$ years; mean $\pm \mathrm{SD}$, duration $15.5 \pm 5.1$ years) and in 12 age-matched healthy male control subjects. Multi-channel frequency analysis of electroencephalogram (electrophysiologic brain mapping) and recording of $\mathrm{P} 300$ and somatosensory evoked potentials were performed before, during and immediately after the hypoglycaemic period. The hypoglycaemia produced a significant increase in low frequency electroencephalographic activity in both groups, most pronounced over anterior regions of the brain. The electroencephalographic activity was normalised immediately after the hypoglycaemic
\end{abstract}

period. The patients with diabetes showed somewhat longer P300 latencies during the initial normoglycaemic examination. Hypoglycaemia caused a marked reduction of the P300 amplitude in both groups of subjects and the amplitude was not restored immediately after normalisation of blood glucose levels. The somatosensory cortical responses were not affected by hypoglycaemia. We conclude that hypoglycaemia results in impairment in cerebral function, as measured by neurophysiological techniques, which is not immediately normalised when blood glucose is restored to normal.

Key words: Electroencephalogram, P300, somatosensory evoked response, hypoglycaemia, diabetes mellitus.
Recurrent hypoglycaemic episodes are common in insulin-treated diabetic patients [1]. Since glucose is the obligate substrate for cerebral metabolism, profound hypoglycaemia may cause permanent brain damage [2-5] and even death $[6,7]$. There is a gradual cerebral deterioration with falling blood glucose levels and electroencephalographic (EEG) studies have shown impairment of brain activity even in moderate hypoglycaemia [8]. Latency of event-related potentials have been reported to increase even at blood glucose levels that are generally regarded as in the lower range of normoglycaemia [9].

Meticulous control of blood glucose is offered to an increasing number of diabetic patients in an effort to prevent or delay diabetic complications. Near-normalised blood glucose profiles may lead to an increment in the frequency of hypoglycaemia [1]. Furthermore, it may well be that improved metabolic control may alter the recognition of hypoglycaemic symptoms [10], leading to a further risk of cerebral deterioration by recurrent hypoglycaemic episodes. Thus, it has been shown that cognitive performance is affected already at blood glucose levels of around $3 \mathrm{mmol} / \mathrm{l}$, starting with alterations in attention and concentration $[12,13]$.

The aim of the present study was to measure cerebral function during and after hypoglycaemia using quantified EEG, P300 and somatosensory evoked potentials in order to further evaluate the influence of hypoglycaemia. The study was undertaken to compare a group of Type 1 (insulin-dependent) diabetic subjects, all of whom had experienced severe hypoglycaemic episodes, with a group of healthy subjects.

\section{Subjects and methods}

\section{Subjects}

Eight male Type 1 diabetic patients (C-peptide $<0.1 \mathrm{nmol} / \mathrm{l}$ ), age $28.0 \pm 7.4$ years (mean $\pm S D$ ) and with a duration of diabetes of $15.5 \pm 5.1$ years participated in the study. Twelve non-diabetic healthy males (age $26.4 \pm 4.2$ years) served as control subjects and another four healthy males (age $27.3 \pm 1.8$ years) took part in a sham study. The normal subjects were medical students without any family history of diabetes mellitus. All subjects were right handed. No subject took any medication except for insulin. In the diabetic patients, the glycosylated haemoglobin $\mathrm{A}_{\mathrm{lc}}\left(\mathrm{HbA}_{1 \mathrm{c}}\right)$ concentration was $8.1 \pm 0.7 \%$ (normal value $<6.0 \%$ ). All patients had experienced symptomatic hypoglycaemia prior to the study and six had had at least one episode of severe hypoglycaemia during the year preceding the study. None of the diabetic subjects had any clinical or laboratory evidence of autonomic neuropathy demonstrable with the immediate heart-rate response to standing, heart-rate variations to deep breathing or to the Valsalva manoeuver. Informed consent was given by all subjects and the study was approved by the Hospital ethics committee. 
Table 1. Test duration and venous blood glucose concentration before, during and after hypoglycaemia

\begin{tabular}{llll}
\hline & \multicolumn{2}{l}{ Type 1 } & \\
\cline { 2 - 4 } & $\begin{array}{l}\text { Diabetic } \\
\text { patients } \\
(n=8)\end{array}$ & $\begin{array}{l}\text { Control } \\
\text { subjects } \\
(n=12)\end{array}$ & $\begin{array}{l}\text { Sham } \\
\text { subjects } \\
(n=4)\end{array}$ \\
\hline $\begin{array}{l}\text { Before } \\
\text { Duration (min) }\end{array}$ & $27(19-40)$ & $27(17-37)$ & $25(15-35)$ \\
$\begin{array}{l}\text { Blood glucose } \\
\text { (mmol/1) }\end{array}$ & $6.4(4.2-7.8)$ & $4.8(4.6-5.1)$ & $4.5(3.7-5.9)$ \\
$\begin{array}{l}\text { During } \\
\text { Duration (min) }\end{array}$ & $35(25-56)$ & $23(16-38)$ & $23(18-26)$ \\
$\begin{array}{l}\text { Blood glucose } \\
\text { (mmol/1) }\end{array}$ & $1.8(1.6-2.2)$ & $1.8(1.6-2.3)$ & $4.3(3.9-5.0)$ \\
$\begin{array}{l}\text { After } \\
\text { Duration (min) }\end{array}$ & $25(18-35)$ & $24(17-35)$ & $22(20-30)$ \\
$\begin{array}{l}\text { Blood glucose } \\
\text { (mmol/1) }\end{array}$ & $6.3(5.3-7.4)$ & $6.2(5.1-7.4)$ & $4.2(3.9-4.6)$ \\
\hline
\end{tabular}

Values are given as medians (ranges within parentheses)

\section{Protocol}

The patients used their ordinary insulin regimens on the day before the study. The experimental tests were carried out after an overnight fast and with the subjects in a sitting position until the studies were completed. An antecubital venous catheter for infusion of insulin and glucose was inserted. A second antecubital catheter was used for continuous venous glucose measurement using a Gambro glucose analyser (Gambro, Lund, Sweden). Venous blood samples were drawn from a hand vein catheter at 5-10 min intervals for immediate measurement of glucose using a Reflolux II glucose meter (Boehringer, Mannheim, FRG) and for later analysis of blood glucose levels by a standard glucose oxidase method, which represent the data given in Results. A constant infusion of regular insulin $\left(2.5 \mathrm{mU} \cdot \mathrm{kg}^{-1} \cdot \mathrm{min}^{-1}\right.$; Actrapid Human, Novo Industries, Denmark) was administered to reach the target blood glucose level of $1.8-2.0 \mathrm{mmol} / \mathrm{l}$ and a graded $20 \%$ glucose infusion was used to maintain this level, and to restore normoglycaemia. During each glucose level, neurophysiological tests were carried out. An identical protocol was used in the sham study, but insulin and glucose infusions were replaced by a continuous infusion of $0.9 \% \mathrm{NaCl}$.

\section{Neurophysiological tests}

The electroencephalogram (EEG) was quantified and frequency analysed (Fast Fourier Transform analysis; FFT) with Biologic Brain Atlas III system. Nineteen channels were recorded monopolarly with linked ear reference. The gain was 30000 to 50000 , the high pass filter $0.3 \mathrm{~Hz}$, the low pass filter $30 \mathrm{~Hz}$, and the sampling frequency of the FFT analysis $128 \mathrm{~Hz}$. The automatic artifact rejection and the $50 \mathrm{~Hz}$ notch filter functions were enabled. The EEG was continuously monitored on paper throughout the sampling periods. Repeated samples of $16 \mathrm{~s}$ EEG were made and the most artifact-free sample was selected for the final analysis.

Somatosensory evoked potentials were recorded with the same equipment and recording electrode montages and the low pass filter changed to $500 \mathrm{~Hz}$ and without the $50 \mathrm{~Hz}$ notch filter. The right median nerve was stimulated with a constant current stimulator and the cathode proximally. The stimulus strength was adjusted to 1.5 times the threshold for muscle twitches in the thenar muscles. The stimulus frequency was $1 \mathrm{~Hz}$ with an interstimulation variance of $10 \%$. Averages of 100 stimuli were made. The analysis time was $128 \mathrm{~ms}$ with a
$25 \mathrm{~ms}$ pre-stimulation period in order to define the baseline. The automatic artifact rejection function was enabled.

P300 responses were recorded by presenting two types of auditory stimuli; Type 1: $2000 \mathrm{~Hz}$ and Type 2: $500 \mathrm{~Hz}$ tone bursts of $100 \mathrm{~ms}$ duration and 80 decibel intensity presented at $1 \mathrm{~Hz}$ with the two stimuli randomly mixed in a 4:1 ratio. The subjects were asked to count the unfrequent type 2 stimuli during the stimulation sessions. The analysis time was $512 \mathrm{~ms}$ with $50 \mathrm{~ms}$ pre-stimulus time. The artifact rejection and notch filter functions were enabled. Sixty samples of type 2 stimuli were averaged.

\section{Statistical analysis}

Effects of hypoglycaemia were evaluated using the paired $t$-test. Group differences were evaluated with the $t$-test with pooled estimates of variance. The sham study was performed to control for the stability of the other measurements and was not used in the statistical evaluation.

\section{Results}

During the period of hypoglycaemia, the target blood glucose concentration was well approximated in both experimental groups (Table 1).

\section{$E E G$}

No major EEG abnormality was found in any subject prior to hypoglycaemia. In Figure 1, the changes in theta activity are shown for the anterior part of the brain. Right and left quadrants are treated together, since no major asymmetry was observed. Similar changes were found in the delta frequency band and for the posterior parts of the brain (data not shown).

A detailed topographical $t$-test analysis of the FFT data, comparing the pre-hypoglycaemic and hypoglycaemic recording in each of the two study groups is illus-

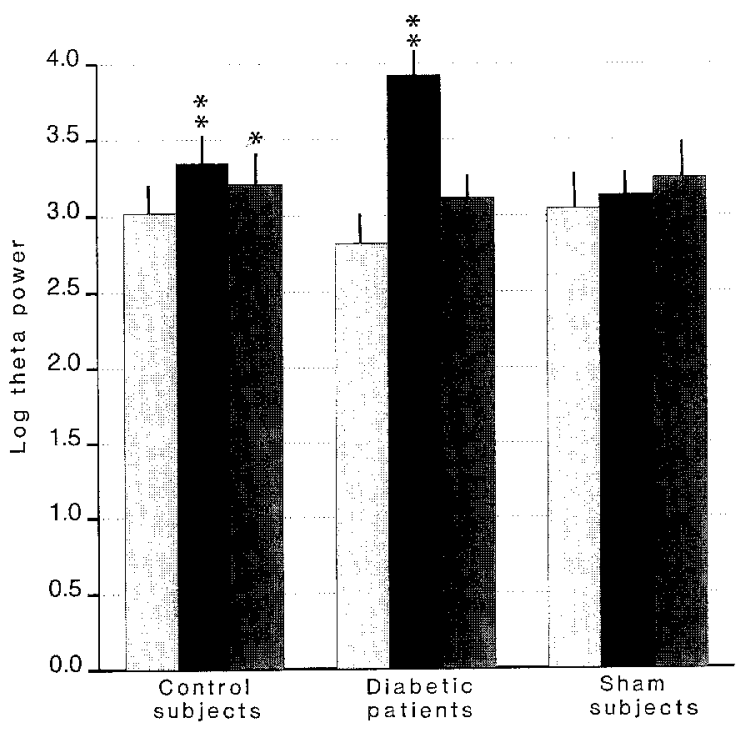

Fig. 1. Theta power $(4.5-8 \mathrm{~Hz})$ in anterior parts of the brain before (white bars), during (black bars) and after (grey bars) hypoglycaemia. The sham group is shown for comparison. The values are given as mean \pm SEM. $*=p<0.05 ; * * *=p<0.01$ (vs prehypoglycaemia) 
A Control subjects
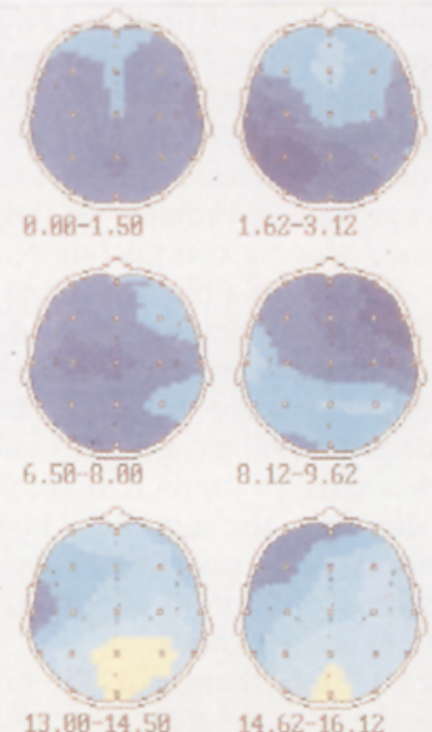

$8.12-9.62$

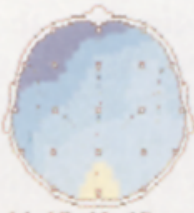

$14.62-16.12$

B Diabetes mellitus

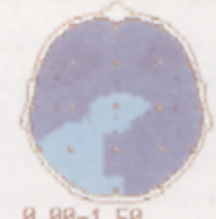

$8.88-1.58$
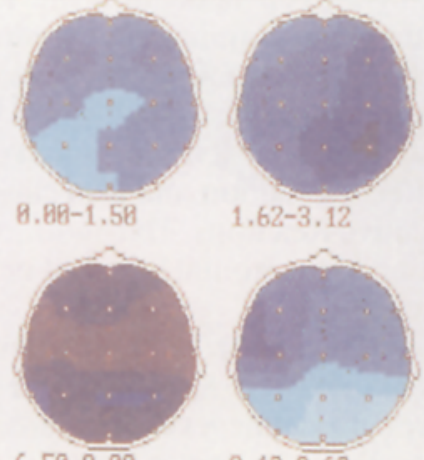

$6.59-8.8 \overline{8}$

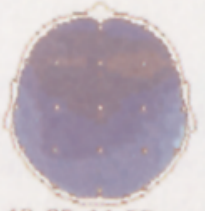

$13.80-14.58$
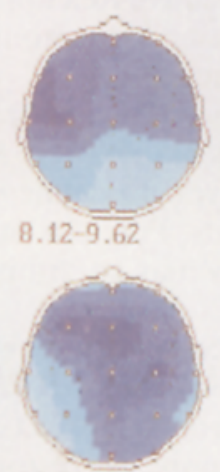

$14.62-16.12$
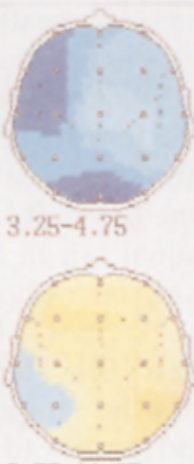

$9.75-11.25$

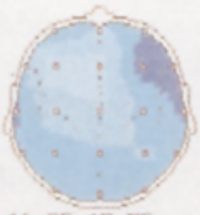

$16.25-17.75$

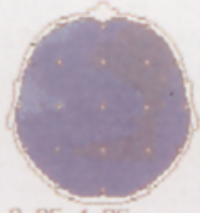

$3.25-4.75$
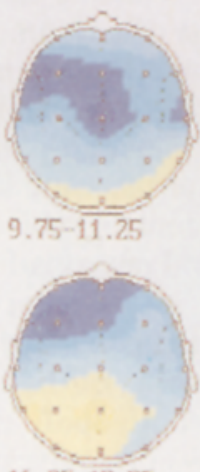

$16.25-17.75$
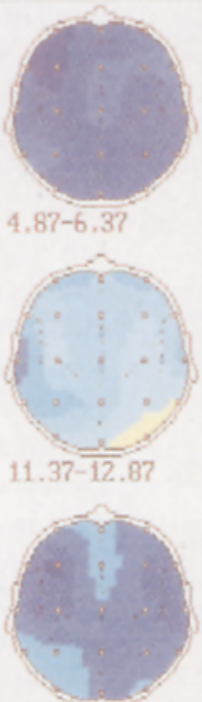

17.87-19.37 Hz
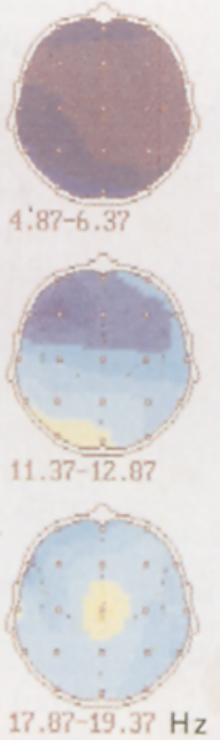

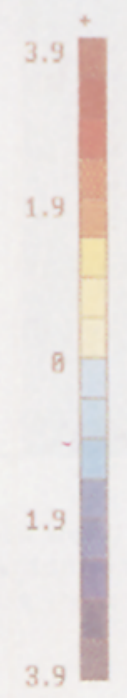

.9

Fig.2. The composite means of $t$-test analysis of the quantified and frequency analysed electroencephalogram (FFT-EEG) data displayed topographically for consecutive frequency bands of the FFT spectra. See text for detailed information trated in Figure 2. The $t$-values indicating an increase of EEG activity within the automatically selected frequency bands during hypoglycaemia are displayed by blue colours, and decrease of EEG activity is indicated by yellow colour. Both groups showed a widespread increase of delta and theta activity $(0.00-8.00 \mathrm{~Hz})$ and an increase of low frequency alpha activity $(8.12-9.62 \mathrm{~Hz})$ anteriorly over the brain. Both groups also showed a decrease of high frequency alpha activity $(9.75-12.87 \mathrm{~Hz})$ and beta activity $(13.0-19.37 \mathrm{~Hz})$ over posterior regions. The increase of theta activity and the anterior release of alpha activity during hypoglycaemia was more marked in the diabetic group compared with the control group.
In the recovery period with normal blood glucose values following hypoglycaemia, the EEG pattern returned to normal. No changes in EEG activity were noted in the sham treated group.

\section{P300}

The diabetic patients had longer P300 latencies prior to hypoglycaemia compared to the control subjects ( $343 \pm 27$ vs $325 \pm 27 \mathrm{~ms}$; mean $\pm \mathrm{SEM} ; p<0.05$ ). In both study groups, a highly significant reduction $(p<0.01)$ in $\mathrm{P} 300$ amplitude was noted during hypoglycaemia (Fig.3). Fur- 


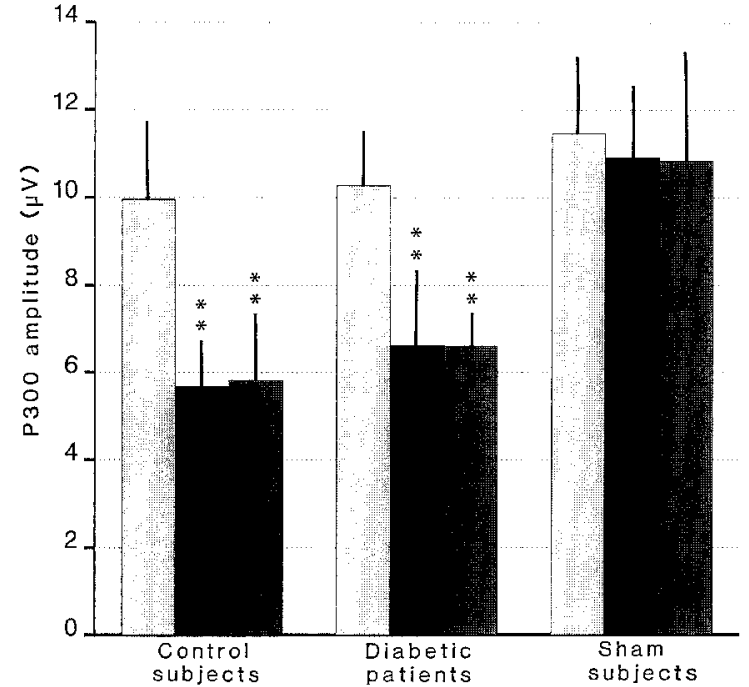

Fig.3. P300 amplitude recorded at Cz before (white bars), during (black bars) and after (grey bars) hypoglycaemia. The sham group is shown for comparison. The values are given as mean $\pm S E M$. $* *=p<0.01$ (vs prehypoglycaemia)

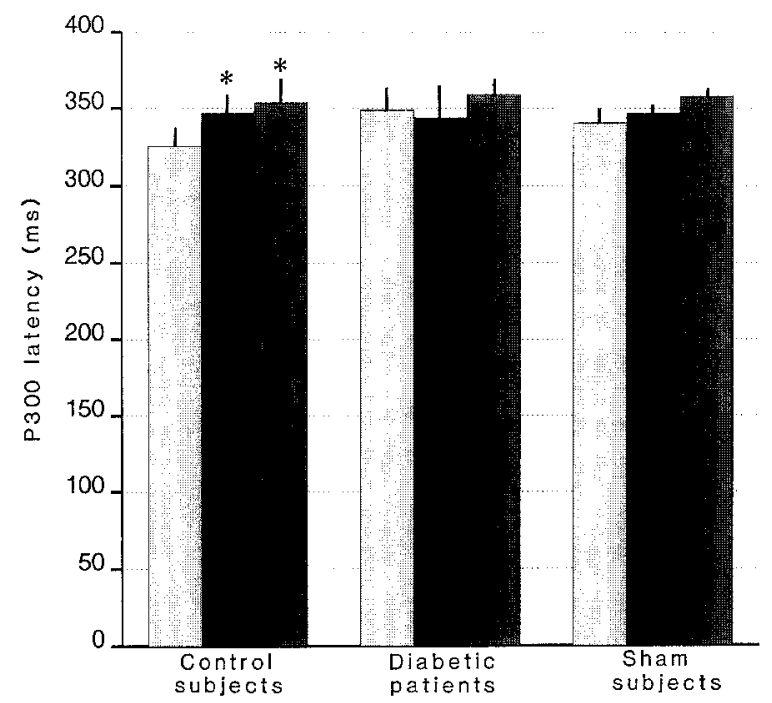

Fig.4. $\mathrm{P} 300$ latency recorded at $\mathrm{Cz}$ before (white bars), during (black bars) and after (grey bars) hypoglycaemia. The sham group is shown for comparison. The values are given as mean \pm SEM. $*=p<0.05$ (vs prehypoglycaemia)

thermore, after return to normoglycaemia following hypoglycaemia, no immediate restitution of the P300 amplitude occurred. No changes were noted in the sham treated group. P300 amplitude changes are shown in Figure 3 for the centrally placed $\mathrm{Cz}$ electrode. Almost the same changes were noted more posteriorly and anteriorly $(\mathrm{Pz}$ and $\mathrm{Fz}$, respectively; data not shown). The P300 latencies did not change in the diabetic patients during hypoglycaemia. In the control group, however, hypoglycaemia resulted in increasing latencies of the P300 wave (Fig. 4).

\section{Somatosensory evoked potentials}

There were no changes in the somatosensory evoked potentials in terms of amplitudes or latencies before, during or after hypoglycaemia (data not shown).

\section{Discussion}

The present study clearly demonstrates neurophysiological changes during hypoglycaemia with a blood glucose level often seen in patients treated with insulin. A substantial increase in low frequency rhythms occurs in the EEG at a blood glucose concentration of around $1.8 \mathrm{mmol} / \mathrm{l}$. These changes are diffusely distributed over the cerebral cortex, but seem to be more pronounced over the anterior parts of the brain. It has previously been shown that during a gradual fall in blood glucose concentration, EEG changes appear rather abruptly at a blood glucose level close to $2.0 \mathrm{mmol} / 1$, with further deterioration at even lower levels [13]. These changes were slightly more pronounced in the temporal and parieto-occipital area. Experimental studies of neuropathological lesions induced by hypoglycaemia show a selective brain damage occurring in a rostro-caudal direction, the superficial layers of the cerebral cortex being one of the selectively vulnerable areas [2-4]. Regional cortical differences of vulnerability within different brain areas are not known.

Most of the significant EEG changes in the present study were found within the anterior areas which is of interest considering the neuropsychological effects of hypoglycaemia. It is generally agreed upon that the frontal lobes play an important role in planning and controlling action [14]. Neuropsychological studies have revealed a gradual deterioration in performance starting at a blood glucose level close to $3.0 \mathrm{mmol} / 1$ [11]. It seems that cognitive dysfunction first affects attention and also causes an inability to handle executive functions. This indicates the possibility of a dysfunction of the frontal lobe and/or its afferent and efferent connections.

There is no obvious explanation to the observation that the diabetic patients showed more marked EEG changes at the same hypoglycaemic level compared with healthy control subjects. Previous minor hypoglycaemic insults and neuropathy may contribute to this phenomenon.

Moderate hypoglycaemia gives rise to a highly significant lowering of the event-related P300 potential amplitudes in both study groups and no early restitution occurred as normoglycaemia was restored. Prolongation of the P300 latency was noted in the control group, but not in the diabetic patients. This may reflect a greater effort among the diabetic patients to concentrate on the task since they had all experienced hypoglycaemia in the past. The finding of increased baseline P300 latencies in the diabetic subjects may imply some impairment of cerebral function already in normoglycaemia in this group. The auditory $\mathrm{P} 300$ paradigm is frequently used since it is easy to apply and produces an identifiable response component. There has previously been some disagreement as to whether P300 reflects information processing activity evoked by a stimulus or whether it is a sign of a general pre-stimulus state of the subject [15]. The former concept is now generally accepted [16] and thus, $\mathrm{P} 300$ relates incoming sensory information to memory updating, a process implying both perception and cognition of stimuli. The origin of $\mathrm{P} 300$ seems to be the temporo-parietal regions. In younger adults, P300 latency and amplitude are 
negatively related [17]. It seems that in an aging population, P300 latency is prolonged despite normal amplituae and for example in depression, the latency is normal with a reduced amplitude. Yet another pattern which can be seen in dementia and after head injury is increased latency and reduced amplitude [18]. Furthermore, P300 latency increases with increasing memory load. In one study of healthy subjects a decrement in plasma glucose concentration from 4.9 to $4.0 \mathrm{mmol} / \mathrm{l}$ caused a significant increase in P300 latencies and these were not normalised until $30 \mathrm{~min}$ after return to normoglycaemia [9]. In another recent study, however, no consistent increase in P300 latency was found in normal subjects when measured at a blood glucose concentration of $3.7 \mathrm{mmol} / \mathrm{l}$ [19].

Alterations in the $\mathrm{P} 300$ wave are thus encountered in a variety of clinical conditions. Whether the observed decrease of amplitude and delay of latency of the P300 wave are due to a general effect such as inability to sustain adequate concentration and attention, or to a specific alteration in cognitive ability can only be speculated upon, but from neuropsychological studies it seems established that attention is a function found to deteriorate early in neuroglucopoenia [11].

No alterations in somatosensory evoked potential amplitudes or latencies could be demonstrated in this study. Obviously, the hypoglycaemia in the present study was not severe enough to affect the present subjects in the same way as has been reported in animal experiments [20].

Intensified insulin regimens are now commonly used which may lead to an increased frequency of hypoglycaemic incidents. This study clearly demonstrates cerebral abnormalities during insulin-induced hypoglycaemia with slowing of electrocortical activity, prolongation of event-related potential latencies and reduction in amplitude. Some of these changes are not immediately restored as blood glucose levels are normalised. The results further focus on the need for taking the risk for hypoglycaemia into account when intensifying insulin treatment in diabetes mellitus.

Acknowledgements. This study was supported by grants from The Medical Faculty, University of Lund, Swedish Diabetes Federation, Malmö Diabetes Association, The Swedish Society of Medicine, The Hoechst Diabetes Foundation and the Swedish Medical Research Council (grant no. B90-14X-00084-26).

\section{References}

1. Cryer PE (1988) Hypoglycemia and insulin-dependent diabetes mellitus. In: Alberti KGMM, Krall LP (eds) The diabetes annual/4. Elsevier, Amsterdam New York Oxford, pp 272-310

2. Kalimo H, Olsson Y (1980) Effects of severe hypoglycemia on the human brain. Neuropathological case reports. Acta Neurol Scand 62: 345-356

3. Agardh C-D, Kalimo H, Olsson Y, Siesjö BK (1980) Hypoglycemic brain injury. I. Metabolic and light microscopic findings in rat cerebral cortex during profound insulin-induced hypoglycemia and in the recovery period following glucose administration. Acta Neuropathol (Berlin) 50:31-41
4. Auer RN, Olsson Y, Siesjö BK (1984) Hypoglycemic brain injury in the rat. Correlation of density of brain damage with the EEG isoelectric time: a quantitative study. Diabetes 33: 10901098

5. Agardh C-D, Rosen I, Ryding E (1983) Persistent vegetative state with high cerebral blood flow following profound hypoglycemia. Ann Neurol 14: 482-486

6. Paz-Guevara AT, Hsu T-H, White P (1975) Juvenile diabetes mellitus after forty years. Diabetes 24:559-565

7. Deckert T, Poulsen JE, Larsen M(1979) The prognosis of insulin dependent diabetes mellitus and the importance of supervision. Acta Med Scand [Suppl] 624: 48-53

8. Harrad RA, Cockram CS, Plump AP, Stone S, Fennich P, Sönksen PH (1985) The effect of hypoglycemia on visual function: a clinical and electrophysiological study. Clin Sci 69: 673-679

9. De Feo P, Gallai V, Mazzotta G, Crispino G, Torlone E, Periello G, Ventura MM, Santeusanio F, Brunetti P, Bolli GB (1988) Modest decrements in plasma glucose concentration cause early impairment in cognitive function and later activation of glucose counterregulation in the absence of hypoglycemic symptoms in normal man. J Clin Invest 82: 436-444

10. Lager I, Attvall S, Blohme G, Smith U (1986) Altered recognition of hypoglycemic symptoms in Type I diabetes during intensified control with continuous subcutaneous insulin infusion. Diab Med 3: 322-325

11. Pramming S, Thorsteinsson B, Theilgaard A, Pinner EM, Binder C (1986) Cognitive function during hypoglycemia in Type I diabetes mellitus. Br Med J 292: 647-650

12. Holmes CS, Koepke KM, Thompson RG, Gyves PW, Weydert YA (1984) Verbal fluency and naming performances in Type I diabetes at different blood glucose concentrations. Diabetes Care 7:454-459

13. Pramming S, Thorsteinsson B, Stigsby B, Binder C (1988) Glycaemic threshold for changes in electroencephalograms during hypoglycemia in patients with insulin dependent diabetes. $\mathrm{Br}$ Med J 296: 665-667

14. Luria AR (1966) Higher cortical functions in man. Tavistock, London

15. Verleger R (1988) Event-related potential and cognition: a critique of the context updating hypothesis and on alternative interpretation of P300. Behav Brain Sci 11: 343-357

16. Ruchkin DS, Sutton S (1979) CNV and P300 relationships for emitted and for evoked cerebral potentials. Prog Clin Neurophysiol 6: 119-131

17. Polish J (1986) Normal variation of P300 from auditory stimuli. Electroenceph Clin Neurophysiol 65: 236-240

18. Curry SH, Woods DL, Low MD (1986) Applications of cognitive ERPs in neurosurgical and neurological patients. In: McCallum WC, Zapoli R, Denoth F (eds) Cerebral psychophysiology: studies in event-related potential. EEG/EMG [Suppl] 38: 469484

19. Kiss I, Ryan CM, Mitrakou A, Jensen T, Durrant J, Gerrich JE (1989) The effects of experimentally induced hypoglycemia on neuropsychological and electrophysiological indices of cognitive function. J Clin Exp Neuropsychol 11: 77 (Abstract)

20. Agardh C-D, Rosen I (1983) Neurophysiological recovery after hypoglycemic coma in the rat: correlation with cerebral metabolism. J Cereb Blood Flow Metab 3: 78-85

Received: 6 July 1989

and in revised form: 17 January 1990

Dr. C.-D. Agardh

Department of Internal Medicine

University Hospital

S-221 85 Lund

Sweden 